\title{
Long-term culture of human liver tissue with advanced hepatic functions
}

\author{
Soon Seng Ng, ${ }^{1,2,3}$ Anming Xiong, ${ }^{1,2}$ Khanh Nguyen, ${ }^{1,2}$ Marilyn Masek, ${ }^{1,2,4}$ Da Yoon No, ${ }^{1,2,5}$ \\ Menashe Elazar, ${ }^{1,2}$ Eyal Shteyer, ${ }^{6}$ Mark A. Winters, ${ }^{1,2}$ Amy Voedisch, ${ }^{7}$ Kate Shaw, ${ }^{7}$ \\ Sheikh Tamir Rashid, ${ }^{1,2}$ Curtis W. Frank, ${ }^{8}$ Nam Joon Cho, ${ }^{3}$ and Jeffrey S. Glenn ${ }^{1,2}$ \\ 'Division of Gastroenterology and Hepatology, Department of Medicine, 2Department of Microbiology and Immunology, \\ Stanford University School of Medicine, Stanford, California, USA. ${ }^{3}$ School of Materials Science and Engineering, Nanyang \\ Technological University, Singapore, Singapore. ${ }^{4}$ Department of Pathology, Stanford University School of Medicine, \\ ${ }^{5}$ Department of Bioengineering, Stanford University, Stanford California, USA. ${ }^{6}$ Department of Pediatric Gastroenterology \\ and Nutrition, Shaare Zedek Medical Center, Jerusalem, Israel. ${ }^{7}$ Department of Obstetrics and Gynecology, ${ }^{8}$ Department of \\ Chemical Engineering, Stanford University, Stanford California, USA.
}

A major challenge for studying authentic liver cell function and cell replacement therapies is that primary human hepatocytes rapidly lose their advanced function in conventional, 2-dimensional culture platforms. Here, we describe the fabrication of 3-dimensional hexagonally arrayed lobular human liver tissues inspired by the liver's natural architecture. The engineered liver tissues exhibit key features of advanced differentiation, such as human-specific cytochrome P450-mediated drug metabolism and the ability to support efficient infection with patient-derived inoculums of hepatitis $C$ virus. The tissues permit the assessment of antiviral agents and maintain their advanced functions for over 5 months in culture. This extended functionality enabled the prediction of a fatal human-specific hepatotoxicity caused by fialuridine (FIAU), which had escaped detection by preclinical models and short-term clinical studies. The results obtained with the engineered human liver tissue in this study provide proof-of-concept determination of human-specific drug metabolism, demonstrate the ability to support infection with human hepatitis virus derived from an infected patient and subsequent antiviral drug testing against said infection, and facilitate detection of human-specific drug hepatotoxicity associated with late-onset liver failure. Looking forward, the scalability and biocompatibility of the scaffold are also ideal for future cell replacement therapeutic strategies.

Conflict of interest: The authors have declared that no conflict of interest exists.

Submitted: September 22, 2016

Accepted: April 27, 2017

Published: June 2, 2017

\section{Reference information:}

JCI Insight. 2017;2(11):e90853.

https://doi.org/10.1172/jici.

insight.90853.

\section{Introduction}

End-stage liver disease (ESLD) is a major cause of world-wide morbidity and mortality. Liver transplantation is the only available treatment, although there is an increasing gap between the need for liver transplantation or cell replacement therapies and the available donor supply (1). Viral infections, such as from hepatitis C virus (HCV), are important etiologies of ESLD. The availability of engineered human liver tissues would be of great benefit for increasing transplantation options, as well as for potential liver assist devices that could serve as a bridge to transplantation.

The liver is also a key organ for processing drugs, and hepatotoxicity is a major cause of drug failure — often discovered late in the development process, sometimes with dramatic consequences (2). The most potent, or toxic, form of a compound may not be the primary compound but rather one of its metabolites (3). Moreover, a compound's primary in vivo metabolite may vary drastically as a function of the animal species in which it is assessed (4). The ability to accurately predict human-specific drug metabolism and hepatotoxicity is essential for more efficient and safer drug development. Unfortunately, current preclinical animal regulatory testing species are unable to detect human-specific drug metabolites or hepatotoxicity. While cultures of human liver cells might help serve this purpose, soon after their plating into standard 2-dimensional culture, primary human liver cells rapidly lose their features of advanced differentiation, such as the expression of cytochrome P450 (CYP) drug metabolizing enzymes or the ability to support infection with natural human hepatitis viruses (5). Liver cell lines, which are derived from human liver tumors, typically also have lost these key markers of their former differentiated state. 
Over the last decade, certain success has been achieved by spatially controlled plating of hepatocytes with nonparenchymal cells using microtechnology-based processes, although these remain largely 2-dimensional (2D) in nature with limited scale-up potential for cell-based therapies $(6,7)$. In addition, the spatial constraints imposed on cells by precision engineering might potentially impede the ability of liver cells to restore their authentic phenotype in vitro due to an overly simplified miroenvironmental niche. To recapitulate the microenvironment of primary hepatocytes, various 3-dimensional (3D) models have been proposed to promote authentic cell-cell interactions and cell polarity that are essential for hepatic phenotypes (8-11). However, while existing 3D culture systems have shown some progress in extending the functions of primary hepatocytes, the functional lifetime of current systems is still limited for critical applications (e.g., long-term hepatotoxicity assessment), and a 3D culture system with demonstrated multifunctional capabilities for supporting viral infection - as well as hepatotoxicity prediction - remains elusive. Generation of equivalent hepatocytes from stem cell-derived resources has promised to overcome these challenges but is unfortunately still currently limited by insufficient functionality, which impairs its application for many downstream assays (12-14). In our previous study (15), human fetal liver progenitors derived from primary human fetal liver tissue have demonstrated traits of parenchymal cell expansion, as well as prolonged hepatic phenotypes in a $3 \mathrm{D}$ coculture system, supporting the notion that they are potentially an excellent cell source for establishing long-term culture systems with advanced hepatic functions.

Here, we sought to test the hypothesis that culturing mixtures of primary human fetal liver cells within a 3D scaffold designed to recreate key aspects of natural liver architecture might better preserve critical features of advanced hepatic differentiation. For this, we were inspired by the $3 \mathrm{D}$ hexagonally arrayed liver lobules - the functional units of human liver - that collectively make up the human liver organ, and we created a network of interconnected hexagonally arrayed spherical cavities within a biocompatible hydrogel, into which primary human liver fetal cell mixtures were seeded. We coated the scaffold with extracellular matrix (ECM) proteins to facilitate cell attachment upon seeding and subsequent cell growth, as evidenced thus far with tumor-derived liver cells $(16,17)$. The architectural features of the scaffold then enable the cells to self-assemble into a 3D configuration. The resulting cultures of primary human fetal liver cells were able to preserve advanced hepatic functions for extended periods of time (at least 5 months), a length of time that has not been achieved with any of the current in vitro liver models $(18,19)$. Importantly, this engineered human liver tissue provided proof-of-concept determination of human-specific drug metabolism, demonstrated the ability to support infection with human hepatitis virus derived from an infected patient and subsequent antiviral drug testing against said infection, and facilitated detection of human-specific drug hepatotoxicity associated with late-onset liver failure.

\section{Results}

Fabrication of 3D hexagonally arrayed lobular human liver tissues. We utilized the unique architecture of an inverted colloidal crystal (ICC) structure to establish functional liver tissue from freshly isolated human fetal total liver cells (FTLCs) in a highly controlled 3D microenvironment. To achieve a 3D scaffold with high mass transportation properties, we first packed a collection of $140-\mu \mathrm{m}$ diameter polystyrene spheres into a mold, followed by annealing, to promote fusion of adjacent spheres (Figure 1, A and B). Polyethylene glycol (PEG) hydrogel precursor solution was then poured into the interstitial spaces between the spheres and polymerized. The spheres were then dissolved using tetrahydrofuran (THF) yielding a 3D hexagonally arrayed collection of spherical cavities, which communicate with each other at the points where adjacent spheres were previously touching (and where no hydrogel could therefore penetrate) (Figure 1C). The regular arrangement and the interconnectivity of cavities across 4 layers of ICC scaffold were visualized using confocal microscopy facilitated by the incorporation of fluorescein (Supplemental Figure 1; supplemental material available online with this article; https://doi.org/10.1172/jci.insight.90853DS1). To determine the effect of an ECM protein such as collagen-I on the growth of cells seeded into the ICC, we introduced an amine active ligand - N-hydroxysuccinimide - on the PEG scaffold for collagen-I conjugation (Supplemental Figure 2A). HUVECs, an anchorage-dependent cell type, were then seeded into ICCs with (Col-I ICC) or without (naked ICC) collagen-I coating. HUVECs reached $100 \%$ confluence and established an elongated morphology along the surface of the cavities after overnight culture in Col-I ICC (Supplemental Figure 2B). In contrast, HUVECs cultured in naked ICC failed to establish attachment and growth. The ability of Col-I ICC to host cells over multiple tiers of the scaffold was revealed by the formation of confluent EGFP-expressing HUVECs over different focal planes, as visualized by confocal imaging (Supplemental Figure 2C). 
A

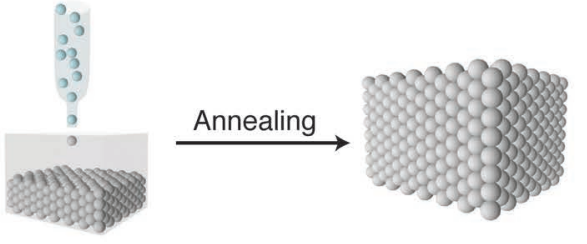

Polystyrene bead deposition
Crystal lattice formation

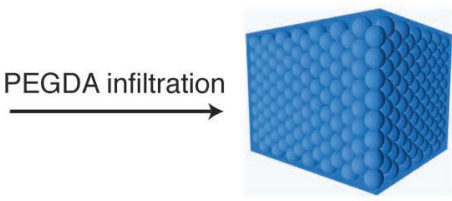

UV polymerization
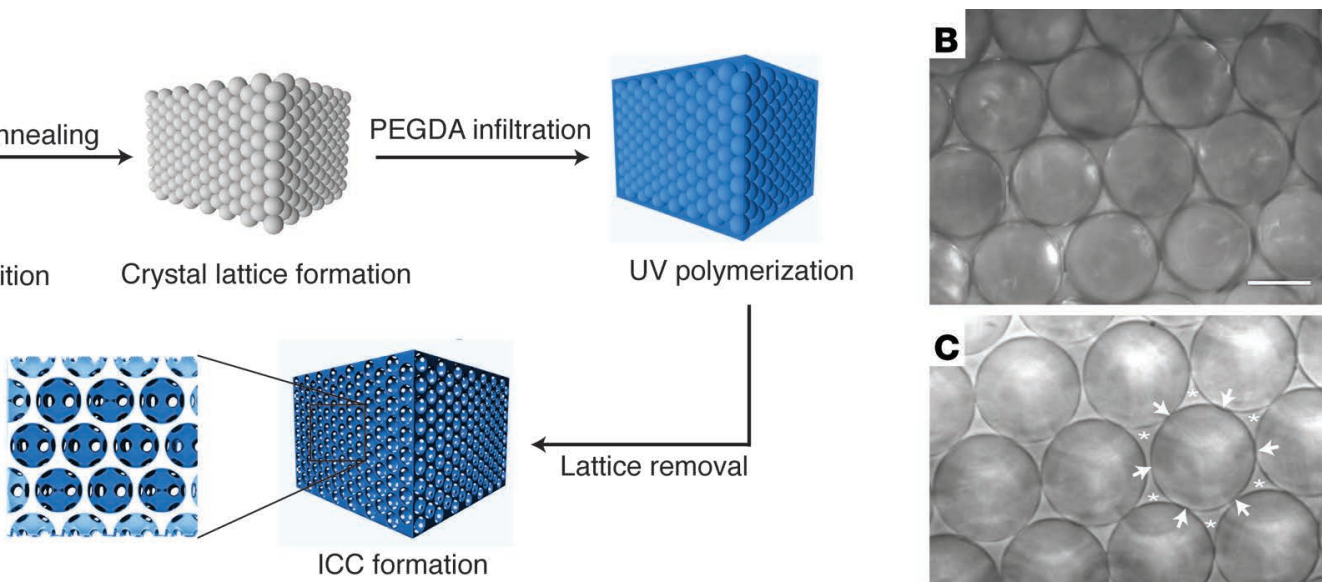

ICC formation
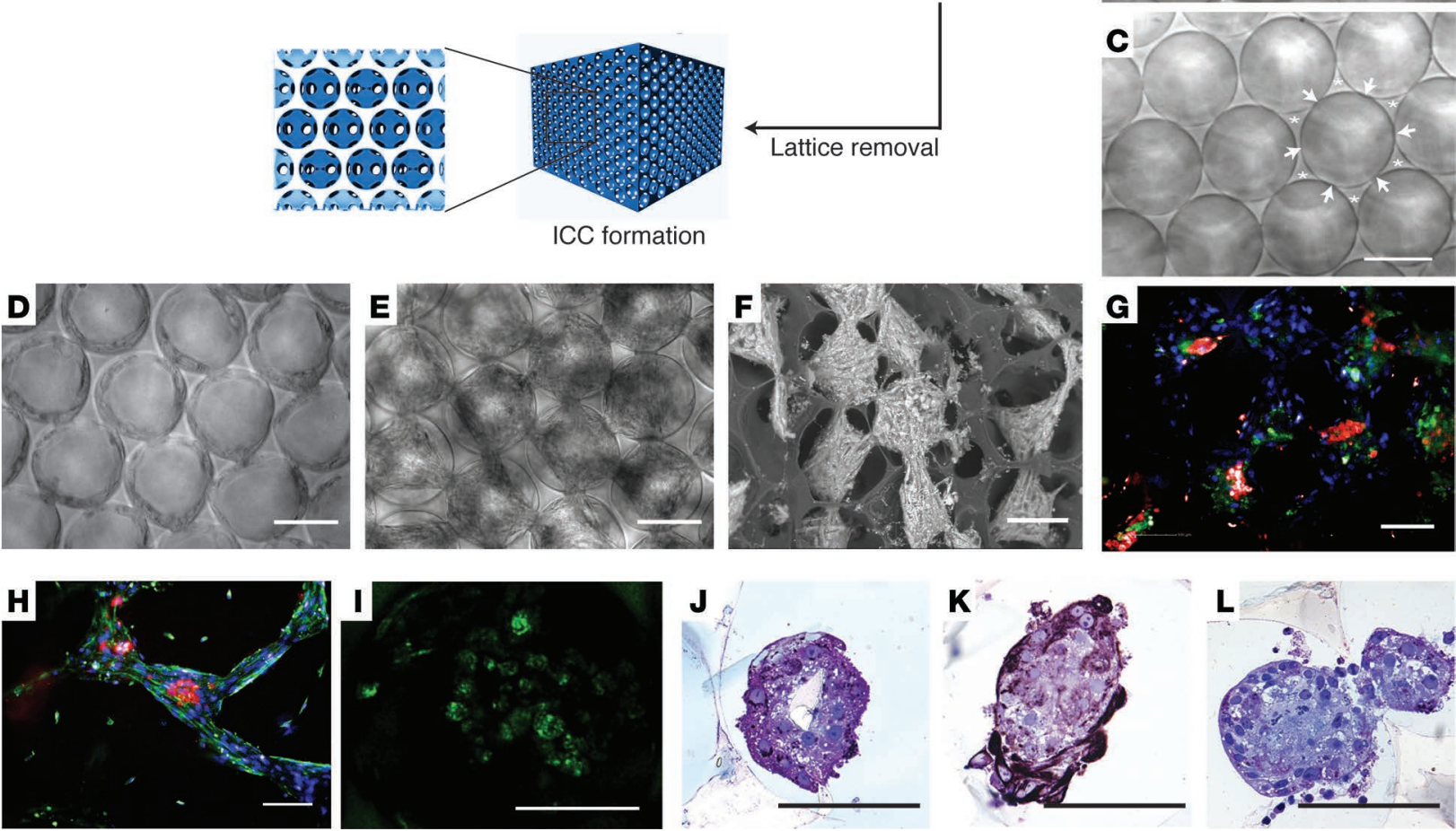

Figure 1. Fabrication of functional 3-dimensional hexagonally arrayed lobular human liver tissues. (A) Schematic illustration of inverted colloidal crystal (ICC) scaffold fabrication. (B) Freestanding colloidal crystal lattice and (C) the resultant ICC with interconnected windows indicated by arrowheads and backbone of ICC indicated by asterisks. Morphology of fetal total liver cells in Col-I ICC (D) upon seeding and (E) 2 weeks after seeding. (F) Variable pressure scanning electron microscopy image demonstrating clusters of liver tissues with high interconnectivity across different ICC tiers. (G and $\mathbf{H})$ Immunofluorescence imaging of engineered liver tissues 2 weeks after seeding for albumin (red) and DAPI (blue) costaining with (G) CK19 (green) or (H) vimentin (green). (I) Accumulation of Cholyl-L-lysyl-fluorescein (CLF) in liver tissues after 40 minutes of CLF incubation, followed by 40 minutes of washing. (J-L) Immunohistological images demonstrating heterogeneous populations within the engineered tissues stained for albumin (J), CK19 (K), and CD68 (L). Scale bars: $100 \mu$ m.

Freshly isolated FTLCs seeded into Col-I ICC demonstrated similar morphology to HUVECs during the initial days of culture (Figure 1D). Upon seeding, FTLCs attached on the surface of cavities and established confluency over the cavity. The attached cells then expanded from the surface toward the cavity centers. At week 2 after seeding, FTLCs self-organized into uniform spheroid-like structures in their respective cavities, with clear connections with adjacent cavities via porous windows (Figure 1E). This morphology remained stable for at least 5 months in culture. Electronic imaging revealed that multicellular liver spheroids were established across different tiers of ICC (Figure 1F). To investigate the differentiation and distribution of cells in these spheroids, we examined the expression of liver epithelial and mesenchymal markers using immunofluorescence staining 2 weeks after seeding. An intense staining of hepatocyte marker, albumin (red), was seen at the core of the spheroid where cells establish strong homotypic cellular interaction (Figure 1, G and $\mathrm{H}$ ). In the same spheroid, cholangiocyte marker CK19 (green, Figure 1G), could often be found in close vicinity to the albumin expressing clusters. Cytoskeletal filaments stained by vimentin (green, Figure $1 \mathrm{H}$ ) were prominently observed over the periphery of the spheroids and the interconnected bridges between cavities. These features indicate a unique self-assembled structure where the liver parenchymal cell clusters were being encapsulated and connected by mesenchymal cells. We also observed cholyl-L-lysyl-fluorescein (CLF) accumulation in engineered tissues after 40 minutes of CLF incubation, followed by immediate 40 minutes of fresh medium incubation (Figure 1I). This observation demonstrated the presence of functional hepatic 


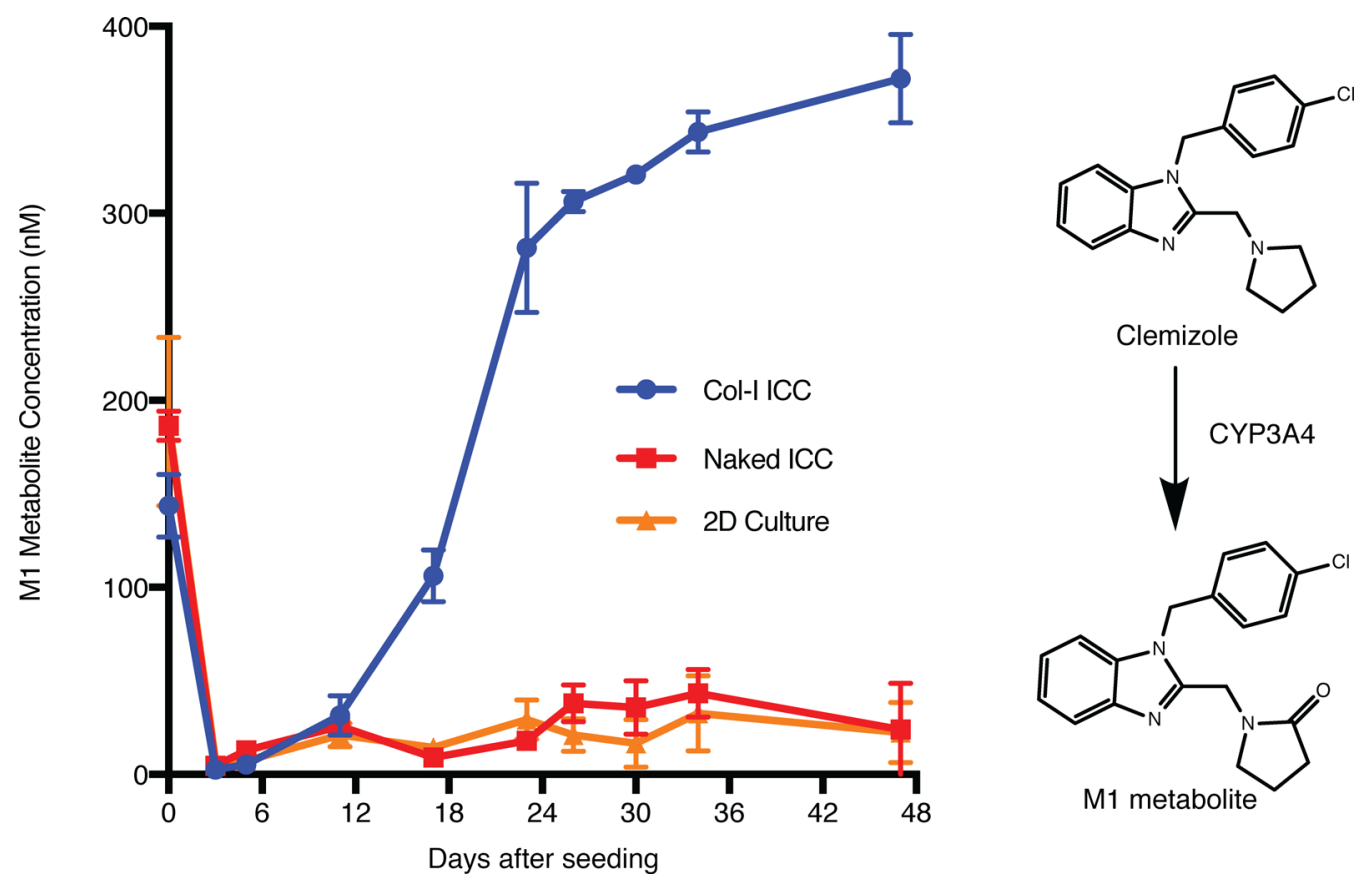

Figure 2. Engineered human liver tissues exhibit prolonged cytochrome P450 drug metabolizing activity. FTLCs in all platforms were treated with $10 \mu \mathrm{M}$ of clemizole at the indicated time points for 24 hours. Supernatants of treated cultures were collected at the end of treatment to measure the production of M1 metabolite using LC/MS. Data are mean \pm SD with at least 5 biological replicates. The right panel shows clemizole's major human metabolite, M1, which is predominantly generated by CYP3A4.

transporters in uptaking and excreting bile salt in the cultured primary liver cells. Further immunohistostainings also revealed the presence of heterogeneous cell populations in the engineered tissues, including albumin (Figure 1J), CK19 (Figure 1K), and CD68 (Figure 1L). Expression of hepatocyte markers could be detected over extensive periods of time (Supplemental Figure 3C)

Prolonged cytochrome P450 drug metabolizing activity in engineered human liver tissues. To investigate the capacity of our engineered human liver tissues to metabolize drugs, we treated our models with different drugs over multiple time points and quantified their respective CYP450-driven production of metabolites using liquid chromatography/mass spectrometry (LC/MS). Clemizole is a first-generation antihistamine that exhibits a high degree of human-specific metabolism characterized by CYP3A4-mediated oxidation to its major human metabolite, M1 (Figure 2) (20). Therefore, M1 metabolite production was used as a surrogate marker of CYP3A4 functional activity. While freshly isolated human FTLCs produced M1, HepG2, a common hepatoma cell line used in conventional cell models for drug discovery, did not (Supplemental Figure 3A). The metabolic function of FTLCs, however, dissipated upon seeding into culture (Figure 2), which is similar to the reported observation with conventional primary liver culture (5). The metabolic function of the FTLCs plated into standard 2D dishes or naked ICCs never recovered. In contrast, FTLCs seeded in Col-I ICC recovered and later exceeded their initial CYP3A4 drug metabolizing activity, reaching a steady state level that was maintained for months (Supplemental Figure 3B). In addition to monitoring CYP3A4 activity, we also assessed the developmental profile of other CYP450 isoforms such as CYP2D6 and CYP2C19 in our engineered human liver tissues. While freshly isolated FTLCs did not demonstrate high CYP2D6 and CYP2C19 activities, the latter increased progressively in Col-I ICC culture and surpassed the initial activity demonstrated by freshly thawed adult hepatocytes (Supplemental Figure 4). All CYP functions of cryopreserved adult hepatocytes in 2D culture dissipated rapidly upon seeding.

Infection with patient-derived HCV inoculum and assessment of antiviral activity. The ability to support efficient infection with natural inoculums of human hepatitis viruses such as HCV represents another feature of advanced human hepatic differentiation characteristic of primary human hepatocytes, which to date cannot be achieved with liver cell lines and is rapidly lost when hepatocytes are plated in 


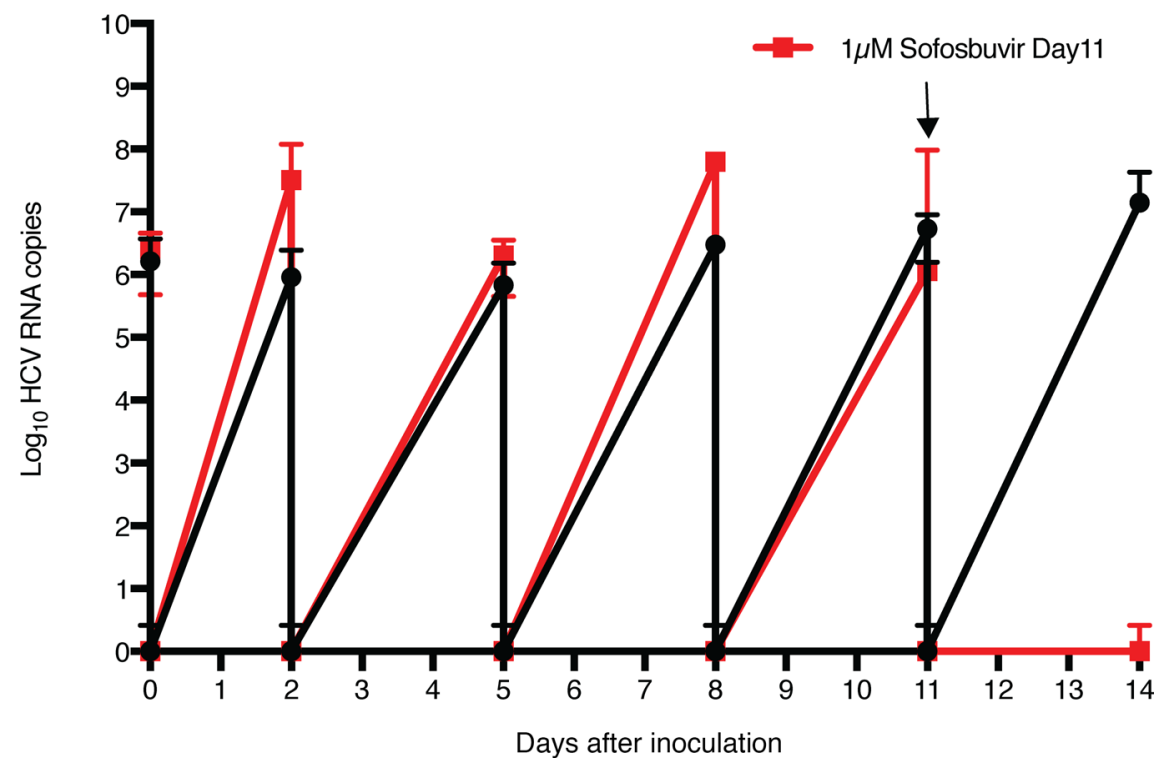

Figure 3. Efficient infection of engineered human liver tissues with patient-derived HCV inoculum and assessment of antiviral activity of sofosbuvir. Parallel cultures of fetal total liver cells (FTLCs) in Col-I ICC were inoculated with hepatitis C virus (HCV) inoculum at day 9 after seeding. At 4 hours after inoculation, virus was removed, cultures were washed 5 times with PBS, and fresh medium was added; samples were collected before and after wash at the indicated intervals for QPCR HCV RNA analysis. One set of cultures was treated with $1 \mu \mathrm{M}$ sofosbuvir at day 11 after inoculation (red tracing; drug treatment indicated by arrow), while another set was treated with vehicle alone (black tracing). Data are mean \pm SD with 5 biological replicates.

standard 2D cultures. To test the ability of the engineered tissues to support infection with authentic human hepatitis virus, an HCV inoculum derived from a patient chronically infected with genotype 1 HCV was used to infect FTLCs cultured in Col-I ICC. The inoculated cultures were washed extensively after inoculation and at each media change to remove residual inoculum or virus released since the preceding media change, respectively. Quantitative PCR (qPCR) demonstrated an efficient infection was established that continuously produced and released HCV virions into the media (Figure 3). No HCV RNA was detected following inoculation of cells from the same source that had been placed into standard 2D culture. To further validate the authentic nature of HCV replication observed in the ICC cultures, and to demonstrate proof of concept for their utility to assess candidate antiviral agents, infected cultures were treated with the nucleoside analog sofosbuvir (21), which successfully abrogated HCV replication to undetectable levels. The ICC cultures could be similarly infected with a HCV genotype 2 a inoculum (Supplemental Figure 5).

Engineered human liver tissues predict fatal human-specific drug hepatotoxicity. To assess the potential of the engineered liver tissues to identify compounds with the potential for serious hepatotoxicity in humans, we determined the ability of the tissues to recapitulate the characteristic human-specific toxicities of fialuridine (FIAU), a nucleoside analog that had cleared all standard preclinical animal safety assessments prior to causing fatal hepatotoxicites associated with lactic acidosis upon subsequent, more extensive clinical testing in humans (22). FTLCs cultured in Col-I ICC for 10 days were incubated in media alone or with increasing concentrations of FIAU. Significant L(+)-lactate was produced by FIAU in a dose-dependent manner following 4 days of treatment (Figure 4A). This phenomenon resembled the elevated serum lactate seen in 7 patients with severe hepatotoxicity due to FIAU treatment in the phase 2 clinical trial (22) and that we recently recapitulated in mice with humanized livers (23). In addition, ultrastructural analyses by transmission electron microscopy (TEM) revealed the signature accumulation of enlarged lipid droplets and a visibly lower number of normal mitochondria in FIAU-treated samples (Figure 4, B-D). Moreover, we also noted significant lactate dehydrogenase $(\mathrm{LDH})$ leakage, deterioration of the rate of albumin production, decreased ATP content and cell viability, and an increase in IL-6 production (Figure 4, E and F). 

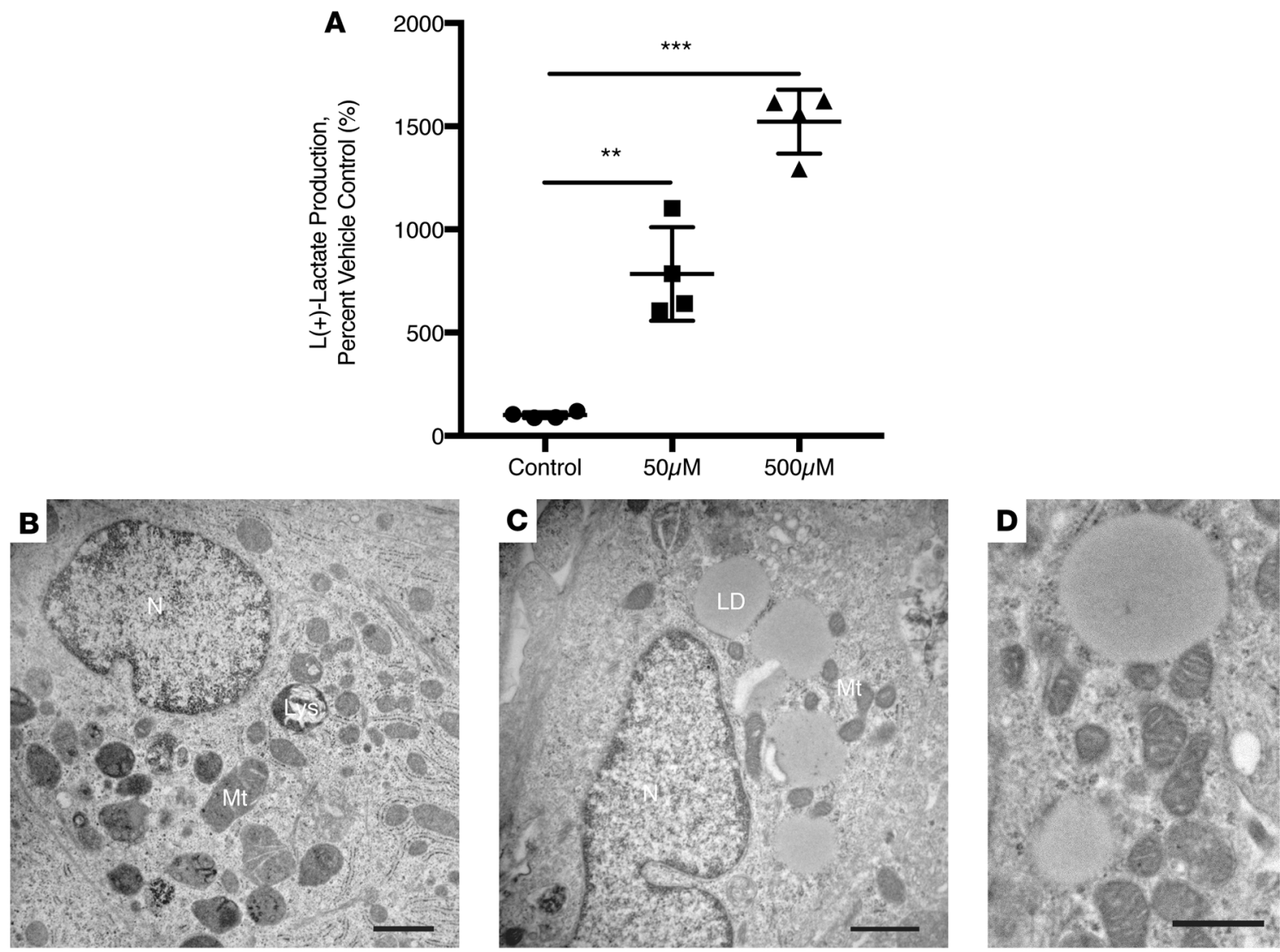

\section{E}

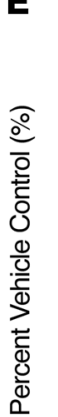
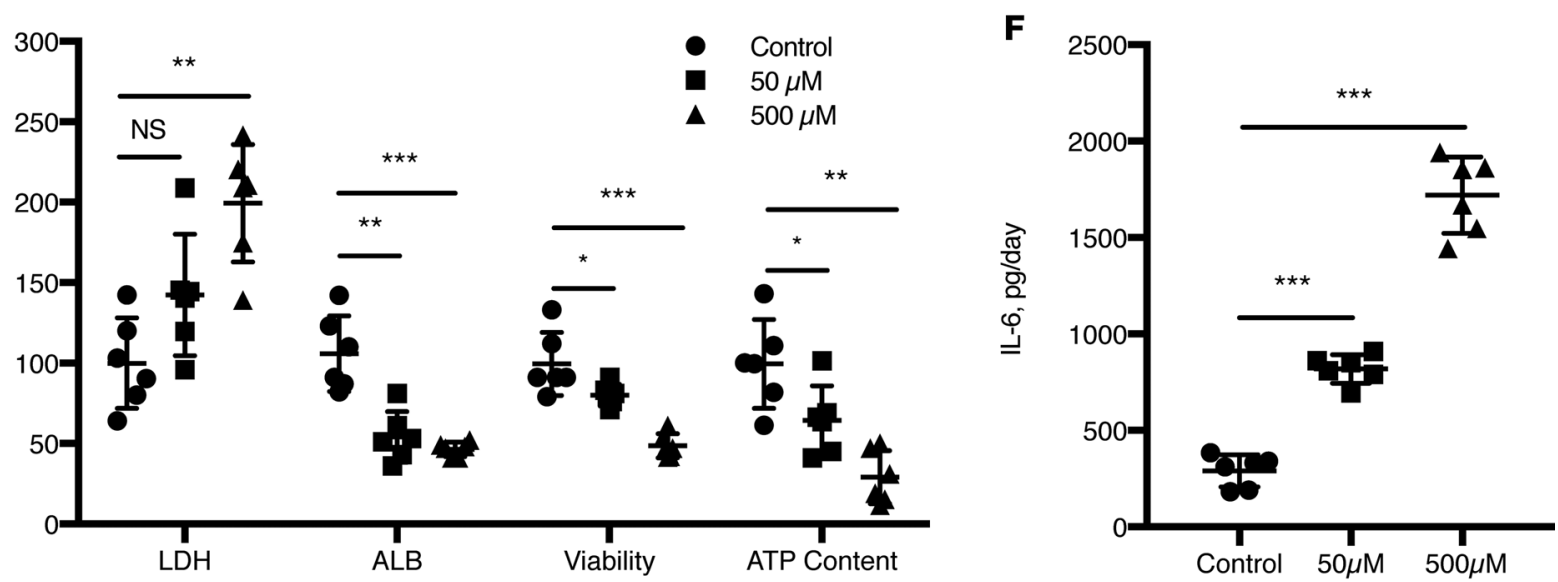

Figure 4. Engineered human liver tissues detect FIAU-induced toxicity associated with fatal human-specific liver injury. Ten-day-old fetal total liver cells (FTLCS) in Col-I ICC were treated with the indicated concentrations of fialuridine (FIAU) or vehicle control for up to 14 days. (A) L(+)-lactate production by FTLCs was monitored in cultures after 4 days of treatment with the indicated concentrations of FIAU $(n=4)$. Ultrastructural images of (B) vehicle only and (C) FIAU-treated hepatocytes with abundant large lipid droplets. N, nucleus; Mt, mitochondria; LD, lipid droplet; Lys, lysosome. Scale bars: $1 \mu \mathrm{m}$. (D) Higher-power image of FIAU treatment group highlights mitochondrial damage and formation of large lipid droplets. Scale bar: $0.5 \mu \mathrm{m}$. (E) Cytotoxicity (LDH leakage), albumin production, cell viability, and ATP content of the cultures were assessed at the end of 14 days after FIAU treatment $(n=6)$. (F) FIAU-treated cultures demonstrated significant production of IL-6 in a dose-dependent manner following 2 days of FIAU treatment. All data was normalized to their respective vehicle-only controls. Statistical significance was analyzed by one-way ANOVA with Dunnett post test. ${ }^{*} P<0.0332,{ }^{* *} P<0.0002,{ }^{* * *} P<0.0001$.

\section{Discussion}

We have developed a 3D in vitro liver model using primary human liver cells that is capable of sustaining advanced human-specific liver functions for at least 5 months in culture, which to our knowledge is the longest reported length of time for culture systems encompassing primary human hepatocytes and represents 
a significant improvement in broad functional capabilities over current systems (Supplemental Table 1). Indeed, the ICC scaffold provides a 3D hexagonal array of interconnected spherical cavities inspired by the liver's lobular structure, into which primary human FTLCs are seeded and grow into an engineered liver tissue. The tissues exhibit features characteristic of highly differentiated hepatocytes, including CYP-mediated drug metabolism, the ability to support efficient infection with natural inoculums of human hepatitis virus, and recapitulation of human-specific drug hepatotoxicity.

Advantages of the PEG-hydrogel scaffold include biocompatibility and the ability to tune the stiffness of the microenvironment by adjusting the size of monomers and the extent to which the resulting polymers are crosslinked. The PEG chemical composition also allows for a high degree of modification, including the incorporation of biological matrix to modulate various cellular responses (24). Here, we demonstrated that the conjugation of type I collagen matrix to the ICC, which is known to facilitate cellular attachment and growth (25) and influence the phenotypic behavior of tumor-derived model liver cells, greatly facilitated the maturation of liver-specific functions of FTLCs - the first such demonstration for primary human hepatocytes. The presence of ECM also enables us to incorporate a cell selectivity feature in our model such that unwanted nonanchorage cells, such as hematopoietic cells that are abundant in fetal liver, can be easily removed from scaffolds by multiple washings. Unlike conventional 3D cell encapsulation culture, where the cells are spatially confined in a homogeneous environment, the ICC provides a quasi-3D environment where cells are first exposed to the ECM-activated surface to establish confluency over the cavity surfaces. The internal geometry of the ICC then enables spontaneous formation of heterotypic liver spheroid-like growth within each cavity and interconnected cell growth across adjacent cavities in a 3D hexagonally arrayed arrangement reminiscent of the lobule architecture characteristic of human liver (26). This spontaneous morphological transformation suggests that FTLCs in ICC scaffolds demonstrated a high intrinsic self-organizing capacity that might be obstructed in both conventional $2 \mathrm{D}$ cultures and those $3 \mathrm{D}$ cultures with lower degrees of freedom (e.g., bulk hydrogels) than the ICC scaffolds.

We have chosen to use human fetal liver cells to establish engineered liver tissues because they can be readily accessed from numerous sources and because the predominant parenchymal cells of fetal liver are hepatic stem cells and hepatoblasts, which are known to have excellent proliferative capability and the potential of hepatic differentiation in vitro $(19,27,28)$. Indeed, the combination of unique characteristics of fetal liver cells and the ICC scaffold have allowed us to produce a 3D-engineered liver model with prolonged culture and advanced hepatic functionality. In addition, we are conducting more experiments to evaluate whether the advantages provided by the ICC-based culture system are able to enhance or maintain the advanced differentiation state of adult human liver cells. This platform may also enable a wide array of additional refinements that collectively further increase the utility and rapprochement to authentic human liver architecture, which include the incorporation of vascular structures and lobular zonation $(29,30)$. The ICC platform should also be ideal for optimizing and assessing the acquisition of true hepatic phenotype by liver precursor cells, such as induced pluripotent or embryonic-derived cells $(12,31,32)$.

A particularly attractive feature of this model is its ability to stably maintain advanced hepatic functions for extensive periods of time. This can enable studies of compounds whose potential toxicity may take time for their full manifestation. Preservation of authentic drug metabolizing enzymes can also enable better preclinical prediction of human-specific drug metabolism. This is important because a given drug can be preferentially metabolized along completely different pathways in standard laboratory rodents versus humans (20, 33). Unfortunately, the in vitro liver models, to date, fail to effectively support the expression of CYP isoforms for more than 3 weeks in culture due to the inevitable dedifferentiation or aberrant expression (6, 33-36).

The ability to support infection with natural, patient-derived inoculums of human hepatitis viruses offers a functionality that is not possible with current tissue culture cell line-based models of hepatitis virus replication that are restricted to HCV replicons or specific infectious clones (37). This can be particularly useful for phenotypic assessments of patient-derived virus or studying natural entry mechanisms and assessing candidate antivirals seeking to target entry. In addition, as shown in Figure 3, drugs that are designed to be specifically activated in human liver and that cannot be assessed for such efficacy in standard rodent models, such as the monophosphate prodrug nucleoside analog sofosbuvir, can be readily evaluated in the ICC model.

A similar limitation of current standard animal models is highlighted by the problem of human-specific drug toxicities. One of the most dramatic examples is FIAU. This compound had shown significant in vitro promise as an antiviral nucleoside analog to treat hepatitis B virus infections. It passed all standard 
preclinical safety assessments and appeared to be well tolerated at low doses for short periods of treatment in humans. With higher doses and longer dosing regimens, however, severe liver toxicity accompanied by lactic acidosis was encountered that eventually led to half the patients dying or requiring liver transplantation (22). Electron microscopic analysis revealed cytoplasmic fat accumulations and abnormal mitochondria, possibly due to human-specific expression of a mitochondrial nucleoside transporter (38). As shown in Figure 4, our engineered liver model was able to readily recapitulate key features of FIAU toxicity such as lactate production and abnormal electron microscopic findings. Interestingly, we also noted a significant increase in IL-6 production associated with FIAU treatment. Although this has not been previously described, we suspect that it may be due to the presence of Kupffer cells in our FTLC preparations and could be a form of acute-phase defense employed by the liver in response to FIAU-induced toxicity (39). Although tragic, a National Academy of Sciences investigation concluded that the human toxicity of FIAU could not have been predicted with the available preclinical animal safety data (40). The ICC model described here could help fill the critical gap in prospective knowledge of a compound's potential for human liver toxicity.

Finally, we believe that these engineered human liver tissues can provide a new system to study other heretofore difficult-to-model important human liver diseases, such as primary or metastatic liver cancer and liver stage malaria infections, and may prove useful for future liver-assist devices or cell-replacement therapies. In summary, we describe a readily scalable 3D human liver coculture model with highly adaptable components. It is capable of exhibiting key features of advanced human hepatic differentiation for extended periods of time, which should be useful for improving drug discovery and development efforts, improving regulatory assessment of candidate drug safety, and studying normal and pathogenic processes dependent on preserved authentic human liver function.

\section{Methods}

Cells, culture media, and chemicals. HUVECs and HepG2 were purchased from ATCC and were confirmed to be mycoplasma free. FTLCs growth media was composed of DMEM supplemented with insulin, transferrin, selenium (ITS + ) premix (BD Pharmingen), $1 \times 10^{-7} \mathrm{M}$ of dexamethasone, $10 \mathrm{mM}$ of nicotinamide, $0.5 \mathrm{mM}$ of ascorbic acid 2-phosphate, $4 \mathrm{mM}$ of L-glutamine, $0.1 \mathrm{mg} / \mathrm{ml}$ of heparin, $5 \% \mathrm{FBS}, 100 \mathrm{U} / \mathrm{ml}$ penicillin $\mathrm{G}$ and streptomycin, and $20 \mathrm{ng} / \mathrm{ml}$ of epithelial growth factor. All cells were cultured in a humidified, $5 \%$ carbon dioxide, $95 \%$ air incubator at $37^{\circ} \mathrm{C}$. All chemicals and regents were purchased from Sigma Chemical unless otherwise specified.

Fabrication of inverse colloidal crystal hydrogel scaffold. Polystyrene beads with diameters of $140 \mu \mathrm{m}$ (Duke Scientific, $3 \times 10^{4}$ particles per milliliter and $1.4 \%$ size distribution) were used to construct a sacrificial crystal lattice, and in-house synthesized poly (ethylene glycol)-diacrylate (PEGDA), acrylate-PEG-N-hydroxysuccinimide (Laysan Bio Inc.) and photoinitiator (Irgacure 2959, BASF) were mixed to fabricate ICC. For this, a suspension of polystyrene beads in $70 \%$ ethanol was seeded into a holder made by cutting off and mounting the top of a 6-mm polypropylene cylindrical tube on to a glass slide with Gorilla Glue (The Gorilla Glue Company). The bead suspension was agitated periodically using an ultrasonic bath to achieve maximal hexagonal arrangement in the holder. The bead suspension was left overnight at room temperature to dry out slowly. The whole complex was heat treated at $120^{\circ} \mathrm{C}$ for 10 hours to anneal the beads. As the result of the annealing process, the surface of individual beads was partially melted and fused with adjacent beads to establish a freestanding colloidal crystal structure. Polyethylene-diacrylate (PEG-DA) was synthesized as previously described (41). In brief, anhydrous PEG powder was dissolved in tetrahydrofuran (THF) at $50^{\circ} \mathrm{C}$. Next, a molar excess of acryloyl chloride with freshly distilled triethylamine was added to the PEG solution and reacted for 5 hours under nitrogen atmosphere. The solution was then allowed to cool to room temperature and recrystallized with diethylether at $4^{\circ} \mathrm{C}$. Lastly, PEG-DA product was purified by a second recrystallization step in fresh anhydrous THF and collected as dry product in a desiccator overnight (41). The precursor solution of in-house synthesized PEG-DA, acrylate-PEG-N-hydroxysuccinimide (Laysan Bio Inc.), and photoinitiator (Irgacure 2959, BASF) were mixed in deionized water at the respective concentrations of 50,10 , and $1 \mathrm{w} / \mathrm{v} \%$. The precursor solution was infiltrated into the colloidal crystal by centrifugation at $500 \mathrm{~g}$ for 5 minutes. Excessive precursor solution was blotted dry and the constructs put under $75 \mathrm{~W}$ xenon UV light source (Oriel Instruments) with a broad range of wavelengths $(200-2,500 \mathrm{~nm})$ for 10 minutes. Upon UV light exposure, the precursor solution underwent free-radical-induced gelation and became insoluble in water. After the polymerization was complete, the colloidal crystal and hydrogel 
construct was soaked in THF for 4 hours to dissolve the polystyrene beads and remove the colloidal crystal structures. The resulting ICC hydrogel scaffolds were equilibrated in deionized water and stored in at $4^{\circ} \mathrm{C}$ for up to 7 days prior to use. For collagen coating, the ICC hydrogel scaffolds were incubated in $100 \mu \mathrm{g} / \mathrm{ml}$ collagen-I solution at $4^{\circ} \mathrm{C}$ for overnight conjugation followed by multiple washes with PBS.

Isolation of human fetal liver cells. Human fetal livers (14-22 weeks) were purchased from Advanced Biosciences Research Inc. or procured from the Stanford University Hospital and Clinics in accordance with all university, state, and federal regulations. Biliary tree and vascular branches of liver tissue were first removed using a scalpel and tweezers. The liver was then minced into smaller size pieces and digested twice with $0.6 \%$ collagenase IV with $0.03 \%$ DNase I in HBSS for 30 minutes at $37^{\circ} \mathrm{C}$. The digested tissue was filtered through a 70- $\mu \mathrm{m}$ nylon mesh to remove fat and clusters. The filtered cells were then centrifuged twice at low speed with PBS in order to remove hematopoietic cells in the supernatant from the total population. To deplete the fibroblast-like cells in total human fetal liver cells, the cell population was further applied to a Ficoll-Paque gradient and centrifuged (Amersham Biosciences, GE Healthcare) for 30 minutes at $4^{\circ} \mathrm{C}$ at $980 \mathrm{~g}$. The resulting cells were defined as human FTLCs.

TEM analysis. Samples were fixed with a solution containing $2 \%$ paraformaldehyde and $2.5 \%$ glutaraldehyde in a sodium cacodylate buffer. Samples were then post-fixed in $2 \%$ osmium tetroxide and dehydrated using an ethyl alcohol series and propylene oxide. Next, samples were infiltrated with epon resin and embedded, and then thick sectioned and stained with toluidine blue, thin sectioned, and poststained with uranyl acetate and lead citrate. Grids were viewed using a Hitachi 7650 transmission electron microscope.

Immunofluorescence and IHC analysis. ICC samples were fixed overnight in buffered 4\% paraformaldehyde. Samples were treated overnight with 2\% Triton-X100 (Sigma-Aldrich) in PBS at room temperature. Samples were first rinsed with PBS then treated with $0.3 \%$ hydrogen peroxide for 5 minutes. Samples were subsequently blocked with 2\% Triton-X100 and 10\% FBS for 1 hour and then incubated with primary antibody (against albumin [Bethyl, A80-129A], cytokeratin 19 [Abcam, ab52625], or vimentin [Abcam, ab24525]) overnight at $4^{\circ} \mathrm{C}$. Samples were washed with PBS and incubated in secondary antibody (Alexa Flour 647 donkey anti-goat IgG [Abcam, ab150131], Alexa Flour 594 donkey anti-rabbit IgG [Abcam, ab150076], or FITC donkey anti-chicken IgY [Abcam, ab63507]) overnight at $4^{\circ} \mathrm{C}$ followed by visualization with a confocal imaging microscope (Operetta, Perkin Elmer) fitted with large-format sCMOS camera and Harmony high content analysis software (Perkin Elmer).

In vitro drug metabolism studies. The primary compounds and their respective major metabolites were identified and characterized by MS (Agilent Technologies) equipped with electrospray ionization source, essentially as described (23). Briefly, the heated capillary temperature in the source was held at $325^{\circ} \mathrm{C}$. Full scan (m/z 110-1,000) spectra or data-dependent MS/MS spectra were collected. The metabolites were identified on the basis of their collision-induced dissociation behavior in MS/MS, accurate mass, and retention time. Quantitative analysis of compounds was performed using a calibration curve at $1,000 \mathrm{ng} / \mathrm{ml}$ of an internal standard 1-(p-bromobenzyl)-2-(1-pyrrolidinylmethyl)-benzimidazole.

$H C V$ infection with sera from HCV-infected patients. Patient sera were filtered and concentrated using a centrifugal filter unit with a pore size of 100,000 nominal molecular weight limit (Merck Milipore). The titer of HCV was determined by qPCR. Total RNA was extracted and purified by TRIzol RNA Isolation reagents (Invitrogen) as per the manufacturer's instructions. First-strand cDNA was synthesized using High-Capacity RNA-to-cDNA Kit (Invitrogen). HCV RNA copy number was quantified using iTaq Universal Probes One-Step Kit (Bio-Rad). The 5' - CTTCACGCAGAAAGCGTCTA - 3' and 5' - CAAGCACCCTATCAGGCAGT - 3' primers were used to amplify HCV RNA, and 6-FAM-TATGAGTGTCGTGCAGCCTC-MGB-NFQ (Applied Biosystems) was used as internal probe. Real-time measurement of PCR products was carried out with CFX96 Real-Time System, C1000 Touch Thermal Cycler (Bio-Rad). The J6/JFH HCVcc genotype 2a clone was a gift from Charles Rice, Rockefeller University, New York, New York, USA.

FIAU-induced hepatotoxicity assays. Cytotoxicity was measured by LDH leakage assay (CytoTox-ONE Homogeneous Membrane Integrity Assay, Promega). Production of L(+)-lactate was monitored using a commercial assay kit according to the manufacturer's (Abcam) instructions. Hepatic-specific function was measured by albumin ELISA assay (Bethyl). Inflammatory cytokine was measured by IL-6 human ELISA kit (Invitrogen). Cell necrosis and mitochondrial function were measured using Mitochondrial ToxGlo assay (Promega) at the end of treatment. 
Statistics. Data were analyzed using Prism 6 software (GraphPad Sofware Inc.) and displayed in mean \pm SD. Pairwise comparisons between experimental and control groups were made using paired or unpaired 2-tailed Student's $t$ test as appropriate. The variance between comparison groups was verified to be equivalent. Multiple group comparisons were made using one-way ANOVA followed by Dunnett's post test. Sample size was preestimated from previously published research and from pilot experiments performed in the laboratory. Unless otherwise stated, $P<0.05$ was considered to be statistically significant.

Study approval. HCV-infected patient serum samples were collected under a research protocol that was approved by the Stanford University Human Subject Research committee (IRB 13859). All samples were collected from patients who provided signed informed consent according to IRB guidelines.

\section{Author contributions}

SSN, ME, CWF, NJC, and JSG developed the study concept and design. SSN, AX, KN, MM, DYN, ES, MAW, AV, STR, and KS acquired data. SSN, KN, ME, CWF, NJC, and JSG analyzed and interpreted the data. SSN, CWF, NJC, and JSG drafted the manuscript and revised the manuscript for intellectual content. NJC and JSG supervised the study.

\section{Acknowledgments}

This research was supported by a Burroughs Wellcome Fund Clinical Scientist Award in Translational Research (JSG); National Research Foundation (NRF-NRFF2011-01) and the National Medical Research Council (NMRC/CBRG/0005/2012) (NJC); the National Institutes of Health R01AI099245 (JSG) and U19AI109662 (JSG); and a Fulbright graduate study award (DYN). This work was supported by the National Research Foundation of Singapore through a Competitive Research Programme grant (NRF-CRP10-2012-07).

Address correspondence to: Jeffrey S. Glenn, Division of Gastroenterology and Hepatology, Department of Medicine, Stanford University School of Medicine, CCSR Building Room 3115 MC 5171, 269 Campus Drive, Stanford, California 94305, USA. Phone: 650.725.3373; E-mail: jeffrey.glenn@stanford.edu. Or to: Nam-Joon Cho, School of Materials Science and Engineering, Nanyang Technological University, RTP-XF06-05A, 50 Nanyang Drive, Singapore 637553, Singapore. Phone: 65.6592.7945; E-mail: njcho@ntu.edu.sg.

1. Klein AS, Messersmith EE, Ratner LE, Kochik R, Baliga PK, Ojo AO. Organ donation and utilization in the United States, 1999-2008. Am J Transplant. 2010;10(4 Pt 2):973-986.

2. Tujios S, Fontana RJ. Mechanisms of drug-induced liver injury: from bedside to bench. Nat Rev Gastroenterol Hepatol. 2011;8(4):202-211.

3. Walgren JL, Mitchell MD, Thompson DC. Role of metabolism in drug-induced idiosyncratic hepatotoxicity. Crit Rev Toxicol. 2005;35(4):325-361.

4. Olson H, et al. Concordance of the toxicity of pharmaceuticals in humans and in animals. Regul Toxicol Pharmacol. 2000;32(1):56-67.

5. Lázaro CA, et al. Establishment, characterization, and long-term maintenance of cultures of human fetal hepatocytes. Hepatology. 2003;38(5):1095-1106.

6. Khetani SR, Bhatia SN. Microscale culture of human liver cells for drug development. Nat Biotechnol. 2008;26(1):120-126.

7. Ploss A, et al. Persistent hepatitis C virus infection in microscale primary human hepatocyte cultures. Proc Natl Acad Sci USA. 2010;107(7):3141-3145.

8. Skardal A, Smith L, Bharadwaj S, Atala A, Soker S, Zhang Y. Tissue specific synthetic ECM hydrogels for 3-D in vitro maintenance of hepatocyte function. Biomaterials. 2012;33(18):4565-4575.

9. Liehl P, et al. Host-cell sensors for Plasmodium activate innate immunity against liver-stage infection. Nat Med. 2014;20(1):47-53

10. Ringel M, et al. Hepatocytes cultured in alginate microspheres: an optimized technique to study enzyme induction. Toxicology. 2005;206(1):153-167.

11. Jitraruch S, et al. Alginate microencapsulated hepatocytes optimised for transplantation in acute liver failure. PLoS ONE. 2014;9(12):e113609.

12. Rashid ST, et al. Modeling inherited metabolic disorders of the liver using human induced pluripotent stem cells. J Clin Invest. 2010;120(9):3127-3136.

13. Kajiwara M, et al. Donor-dependent variations in hepatic differentiation from human-induced pluripotent stem cells. Proc Natl Acad Sci USA. 2012;109(31):12538-12543.

14. Loh KM, et al. Efficient endoderm induction from human pluripotent stem cells by logically directing signals controlling lineage bifurcations. Cell Stem Cell. 2014;14(2):237-252.

15. Xiong A, et al. Isolation of human fetal liver progenitors and their enhanced proliferation by three-dimensional coculture with endothelial cells. Tissue Eng Part A. 2008;14(6):995-1006.

16. Kim MH, Kumar SK, Shirahama H, Seo J, Lee JH, Cho NJ. Phenotypic regulation of liver cells in a biofunctionalized three-dimensional hydrogel platform. Integr Biol (Camb). 2016;8(2):156-166. 
17. Kim MH, et al. Biofunctionalized Hydrogel Microscaffolds Promote 3D Hepatic Sheet Morphology. Macromol Biosci. 2016;16(3):314-321

18. Bhatia SN, Underhill GH, Zaret KS, Fox IJ. Cell and tissue engineering for liver disease. Sci Transl Med. 2014;6(245):245sr2.

19. Palakkan AA, Hay DC, Anil Kumar PR, Kumary TV, Ross JA. Liver tissue engineering and cell sources: issues and challenges. Liver Int. 2013;33(5):666-676.

20. Nishimura T, et al. Using chimeric mice with humanized livers to predict human drug metabolism and a drug-drug interaction. J Pharmacol Exp Ther. 2013;344(2):388-396.

21. Murakami E, et al. Mechanism of activation of PSI-7851 and its diastereoisomer PSI-7977. J Biol Chem. 2010;285(45):34337-34347.

22. McKenzie R, et al. Hepatic failure and lactic acidosis due to fialuridine (FIAU), an investigational nucleoside analogue for chronic hepatitis B. N Engl J Med. 1995;333(17):1099-1105.

23. Xu D, et al. Fialuridine induces acute liver failure in chimeric TK-NOG mice: a model for detecting hepatic drug toxicity prior to human testing. PLoS Med. 2014;11(4):e1001628.

24. Zhu J. Bioactive modification of poly(ethylene glycol) hydrogels for tissue engineering. Biomaterials. 2010;31(17):4639-4656

25. Suzuki A, Iwama A, Miyashita H, Nakauchi H, Taniguchi H. Role for growth factors and extracellular matrix in controlling differentiation of prospectively isolated hepatic stem cells. Development. 2003;130(11):2513-2524.

26. Godoy $\mathrm{P}$, et al. Recent advances in $2 \mathrm{D}$ and $3 \mathrm{D}$ in vitro systems using primary hepatocytes, alternative hepatocyte sources and non-parenchymal liver cells and their use in investigating mechanisms of hepatotoxicity, cell signaling and ADME. Arch Toxicol. 2013;87(8):1315-1530.

27. Turner R, et al. Human hepatic stem cell and maturational liver lineage biology. Hepatology. 2011;53(3):1035-1045.

28. Schmelzer E, et al. Human hepatic stem cells from fetal and postnatal donors. J Exp Med. 2007;204(8):1973-1987.

29. Rouwkema J, de Boer J, Van Blitterswijk CA. Endothelial cells assemble into a 3-dimensional prevascular network in a bone tissue engineering construct. Tissue Eng. 2006;12(9):2685-2693.

30. Gebhardt R, Matz-Soja M. Liver zonation: Novel aspects of its regulation and its impact on homeostasis. World J Gastroenterol. 2014;20(26):8491-8504.

31. Schwartz RE, et al. Modeling hepatitis C virus infection using human induced pluripotent stem cells. Proc Natl Acad Sci USA. 2012;109(7):2544-2548.

32. Yagi H, et al. Embryonic and induced pluripotent stem cells as a model for liver disease. Crit Rev Biomed Eng. 2009;37(4-5):377-398

33. Dash A, et al. Liver tissue engineering in the evaluation of drug safety. Expert Opin Drug Metab Toxicol. 2009;5(10):1159-1174.

34. Hegde M, et al. Dynamic interplay of flow and collagen stabilizes primary hepatocytes culture in a microfluidic platform. Lab Chip. 2014;14(12):2033-2039.

35. Bale SS, et al. Long-term coculture strategies for primary hepatocytes and liver sinusoidal endothelial cells. Tissue Eng Part C Methods. 2015;21(4):413-422.

36. Gerets $\mathrm{HH}$, et al. Characterization of primary human hepatocytes, HepG2 cells, and HepaRG cells at the mRNA level and CYP activity in response to inducers and their predictivity for the detection of human hepatotoxins. Cell Biol Toxicol. 2012;28(2):69-87.

37. Bukh J, Purcell RH. A milestone for hepatitis C virus research: a virus generated in cell culture is fully viable in vivo. Proc Natl Acad Sci USA. 2006;103(10):3500-3501.

38. Lee EW, Lai Y, Zhang H, Unadkat JD. Identification of the mitochondrial targeting signal of the human equilibrative nucleoside transporter 1 (hENT1): implications for interspecies differences in mitochondrial toxicity of fialuridine. J Biol Chem. 2006;281(24):16700-16706.

39. Taub R. Liver regeneration: from myth to mechanism. Nat Rev Mol Cell Biol. 2004;5(10):836-847.

40. Institute of Medicine Committee to Review the Fialuridine Clinical T. In: Manning FJ, Swartz M, eds. Review of the Fialuridine (FIAU) Clinical Trials. Washington (DC): National Academies Press (US); 1995.

41. Myung D, et al. Biomimetic strain hardening in interpenetrating polymer network hydrogels. Polymer. 2007;48:5376-5387. 